\title{
Aging in Place: Estimating the Impact of Unexpected Intensive Care Unit Admission on Long-Term Functional Outcomes of Older Adults after Major Cancer Surgery
}

\author{
Jason T. Wiseman, MD, MSPH ${ }^{1,2,3}$, Carmen Quatman, $\mathrm{MD}^{4}$, and Jordan M. Cloyd, $\mathrm{MD}^{5}$ (1) \\ ${ }^{1}$ Dignity Health Cancer Institute, St. Joseph's Hospital and Medical Center, Phoenix, AZ; ${ }^{2}$ Creighton University School of \\ Medicine, Phoenix, AZ; ${ }^{3}$ University of Arizona College of Medicine, Phoenix, AZ; ${ }^{4}$ Department of Orthopedic Surgery, \\ The Ohio State University Wexner Medical Center, Columbus, OH; ${ }^{5}$ Division of Surgical Oncology, Department of \\ Surgery, The Ohio State University Wexner Medical Center, Columbus, OH
}

Given general epidemiologic trends in aging and advances in perioperative medicine, older patients with cancer are increasingly offered and subsequently undergo major cancer surgery. ${ }^{1}$ Older adults undergoing high-risk cancer surgery are at increased risk for postoperative complications; in turn, these may lead to prolonged recovery, nonhome discharge, long-term functional disability, cognitive decline, and/or death. While there has been increasing emphasis on incorporating patient-reported outcome (PRO) measures for optimization after surgery, older patients in particular place a strong emphasis on quality of life. The biopsychosocial needs of older adults are often very different from those of younger patients, and traditional curative-intent therapeutic goals often need to be tempered with more preference-concordant goals of disease management. ${ }^{2}$ Indeed, "aging in place" is increasingly a primary goal of care for older patients that prioritizes living independently and avoiding the need for long-term or permanent assisted living. ${ }^{3}$ Nevertheless, patient understanding of postoperative risks following major cancer surgery is poor, and there is limited evidencebased shared-decision-making tools that personalize risks of traditional postoperative and patient-reported outcomes, particularly for cancer patients. ${ }^{4,5}$ The impact of

(C) Society of Surgical Oncology 2021

First Received: 4 August 2021

Accepted: 12 August 2021;

Published Online: 26 August 2021

J. M. Cloyd, MD

e-mail: Jordan.Cloyd@osumc.edu postoperative complications on long-term functional outcomes of older cancer surgery patients is poorly understood and yet critically important to informing shared decisionmaking that is concordant with patient preferences.

In this context, Tillmann and colleagues performed a population-based cohort study to evaluate the long-term impact of unexpected intensive care unit (ICU) admission following major cancer surgery among older adults. ${ }^{6}$ Using the Ontario Cancer Registry and several linked Canadian health databases, the authors studied all patients 70 years and older who underwent high-intensity cancer surgery (defined as major pulmonary, gastrointestinal, endocrine, and urological surgery) between the years 2007 and 2017. The primary outcome of interest was "time alive and at home following surgery," measured as time elapsed to nursing home admission or death. Using marginal Cox proportional hazards regression, the authors calculated the association between unexpected ICU admission (defined as any admission after postoperative day 0 or with mechanical ventilation on day of surgery) and long-term outcomes. The models accounted for potential confounders (e.g., patient demographics, comorbidities, frailty index, type and stage of cancer, receipt of neoadjuvant therapy, etc.) and institution-level clustering.

Among the 47,367 older adults undergoing major cancer surgery, $15.6 \%$ experienced unexpected ICU admission. Those who experienced unexpected ICU admission spent less time alive at home (median time 16.2 months, 5-year rate $26.2 \%$ ) compared with those who did not require ICU stay (median time $>5$ years, 5 -year rate $56.8 \%$ ). While patients with unexpected ICU admission were older, had a higher burden of comorbid illnesses, and a higher 
proportion of gastrointestinal malignancy compared with those without unexpected ICU, this association remained significant even on multivariable modeling. Furthermore, this effect was modified by length of mechanical ventilation, with longer ventilator support associated with increased risk. Finally, the independent impact of unexpected ICU stay on the primary outcome was greatest during the first 6 months but nevertheless persisted throughout the measured 5 years.

The authors should be commended on this excellent study that highlights the impact of unexpected ICU admission after cancer surgery on aging in place. These data are striking in that, even after controlling for potentially confounding factors, the association between ICU admission and independent living was not only present in the immediate perioperative period but persisted for years. A highlight of the current study is the focus on patientcentered functional outcomes. The authors were specifically interested in evaluating the ability of patients to maintain their functional independence after surgery and not require admission to a nursing home. While older patients generally prefer to "age in place" because it permits them to maintain their autonomy, independence, and connection to social support, including family and friends, ${ }^{7}$ this metric is also meaningful since loss of independence after surgery in older adults is associated with postoperative complications, readmissions, and death after discharge. ${ }^{8}$ Given this, the endpoint "alive at home" is a clinically relevant and patient-centered outcome important for patients and providers in consideration of how high-risk cancer surgery may ultimately impact goals of care.

An important application of these data is the ability to improve patient counseling and shared decision-making based on patient treatment goals. Thoughtful discussion of the association between unplanned ICU admission, disposition to a nursing home, and risk of death and its importance with patients before and after surgery may lead to an improved understanding of expectations as well as a patient-centered approach to shared decision-making. This study also establishes that the more "intensive" the ICU care (defined by number of days requiring mechanical ventilation), the more likely discharge to a nursing home and/or death during the follow-up period becomes. This information not only facilitates preoperative counselling but also may allow ongoing education regarding a patient's functional prognosis throughout a dynamic postoperative course.

The study by Tillmann et al. has other important implications. First, older patients who survive unexpected ICU admission after high-intensity cancer surgery may represent a high-risk population that could benefit from patient-centered interventions such as rehabilitation, more frequent medical evaluation, palliative care consultation, and/or advance care planning. Second, while patients who experienced unexpected ICU admission were at higher risk for death or admission to a nursing home for up to 5 years following surgery, the majority were able to remain living at home. Furthermore, greater than half of patients with unexpected ICU admission remained alive and at home for over 1 year. Thus, there remains a role for aggressive lifeprolonging therapies among older adults following highintensity cancer surgery. It is noteworthy, as Tillmann and colleagues point out, that cancer patients represent a highly select population that is likely healthier than the average ICU population and the indication for ICU admission in this select population may be related to reversible postoperative events that in turn portend a better chance of recovery.

Despite the strengths of this study, several limitations should be acknowledged. First, the authors present evidence of a strong association between unexpected ICU admission and long-term functional decline but cannot show causation between the two. Indeed, it seems less likely that the need for nursing home admission, outside of the first 3-6 months following surgery, is directly related to a patient's cancer surgery or ICU stay (as opposed to some other health condition). In fact, only $0.8 \%$ of patients were discharged directly from the hospital to a nursing home. Rather, unexpected ICU admission is likely a surrogate, akin to frailty, that identifies patients at higher risk for death or nursing home admission over the next 5 years. Second, the unique structure of the Ontario healthcare system makes direct analogies to other healthcare systems (e.g., in the USA) challenging. While nursing home visits in the USA are often temporary following a medical or surgical illness, the structure of nursing home care in Ontario is designed such that patients who are admitted to a nursing home are not expected to improve to a point where they can be discharged.

In summary, Tillmann et al. present a sophisticated analysis of a population-based cohort of older patients undergoing major cancer surgery and find an increased risk for death or admission to a nursing home for at least 5 years after unexpected ICU admission. Given the importance of maintaining functional independence to older patients, these clinically relevant and patient-centered data will undoubtedly help equip physicians and patients in shared decision-making for complex, high-risk cancer surgery.

DISCLOSURES The authors declare no conflicts of interest.

\section{REFERENCES}

1. Smith BD, Smith GL, Hurria A, Hortobagyi GN, Buchholz TA. Future of cancer incidence in the United States: burdens upon an aging, changing nation. J Clin Oncol. 2009;27(17):2758-65. 
2. Kane RL, Ouslander JG, Resnick B, Malone ML. Essentials of clinical geriatrics. 7e edn. New York: McGraw-Hill; 2013.

3. Healthy Places Terminology. Centers for disease control and prevention. https://www.cdc.gov/healthyplaces/terminology.htm. Published 2021. Accessed 26 July 2021.

4. Schenker Y, Fernandez A, Sudore R, Schillinger D. Interventions to improve patient comprehension in informed consent for medical and surgical procedures: a systematic review. Med Decis Mak. 2011;31(1):151-73. https://doi.org/10.1177/0272989X10364247.

5. Taylor LJ, Nabozny MJ, Steffens NM, et al. A framework to improve surgeon communication in high-stakes surgical decisions best case/worst case. JAMA Surg. 2017;152(6):531-8.

6. Tillman B, Hallet J, Guttman M, et al. A population-based analysis of long-term outcomes among older adults requiring unexpected intensive care unit admission after cancer surgery. Ann Surg Oncol. 2021. https://doi.org/10.1245/s10434-021-10705-8.

7. Wiles JL, Leibing A, Guberman N, Reeve J, Allen RES. The meaning of "aging in place" to older people. The Gerontologist. 2012;52(3):357-66.

8. Berian JR, Mohanty S, Ko CY, Rosenthal RA, Robinson TN. Association of loss of independence with readmission and death after discharge in older patients after surgical procedures. JAMA Surg. 2016;151(9):e161689.

Publisher's Note Springer Nature remains neutral with regard to jurisdictional claims in published maps and institutional affiliations. 